\title{
P02-7-15 Poster session
}

\section{Modulation of DDAH/ADMA/NOS pathway in pancreatic $\beta$-cells under diabetic condition}

\author{
Yukiko K. Kaneko, Misaki Sano, Ami Morioka, Masato Nojiri, Nahoko Kasahara, Tomohisa Ishikawa \\ Department of Pharmacology, School of Pharmaceutical Sciences, University of Shizuoka, Japan
}

Nitric oxide (NO) regulates various physiological and pathophysiological processes. We have previously demonstrated that a small amount of NO, which is produced by constitutive NO synthase (cNOS), has a dual effect on insulin secretion, depending on its concentration. Furthermore, we have previously shown that low-concentration NO, which is assumed to be produced by cNOS, exhibits an anti-apoptotic effect in $\beta$-cells. Thus, the deterioration of cNOS-derived NO production is likely to be involved in the dysfunction of pancreatic $\beta$-cells. Asymmetric $\mathrm{N}^{\mathrm{G}}, \mathrm{N}^{\mathrm{G}}$-dimethyl-L-arginine (ADMA) is an endogenous methylated amino acid that inhibits NOS. ADMA is formed when arginine residues in proteins are methylated by protein arginine methyltransferase (PRMT), and is metabolized to L-citrulline by dimethylarginine dimethylhydrolase (DDAH). The present study investigated the involvement of the DDAH/ADMA/NOS pathway in the regulation of NO production in pancreatic $\beta$-cells under diabetic conditions. The mRNA expression of DDAH and PRMT was determined in the pancreatic $\beta$-cell line INS-1. In endothelial cells, the expression of DDAH2 has been shown to be decreased by 5 -day culture in the presence of high glucose. We thus investigated the effect of high glucose on the expression of DDAH mRNA in INS-1 cells using quantitative PCR. INS-1 cells were cultured in the medium containing 10 or $20 \mathrm{mmol} / 1$ glucose for 3 days. We found no significant differences in the expression of DDAH1 mRNA between the different glucose conditions. In contrast, DDAH2 mRNA expression was strongly reduced in the cells treated with $20 \mathrm{mmol} / 1$ glucose. Moreover, the protein expression of DDAH1 was also unchanged, whereas that of DDAH2 was significantly suppressed in the cells treated with $20 \mathrm{mmol} / \mathrm{l}$ glucose. Finally, we measured ADMA contents in INS-1 cells treated with high glucose by HPLC, and found that the ADMA formation was increased in the presence of $20 \mathrm{mmol} / \mathrm{l}$ glucose. These results suggest that the DDAH/ADMA/NOS pathway is working in $\beta$-cells and might contribute to modulate NO production in pancreatic $\beta$-cells under diabetic conditions, possibly by changing the balance between production and metabolism of ADMA. 